\title{
¿Investigación en Psicoterapia? Claro que sí. Pero, ¿A quién le interesa? ${ }^{1}$
}

\author{
JuanPabloJiménez ${ }^{2}$
}

\section{Resumen}

El presente trabajo es una reflexión crítica sobre el interés que los resultados de investigación en psicoterapia con metodología empírica tiene para:

$1^{\circ}$ Las autoridades de Salud y la industria de seguros de enfermedad, que necesitan planificar la prestación de servicios psicoterapéuticos. En este punto se alerta en contra del uso irreflexivo que las autoridades están haciendo del conocimiento empírico.

$2^{\circ}$ Los psicoterapeutas clínicos que buscan ampliar la base de evidencia de sus técnicas de tratamiento. Aquí se destacan los factores curativos en la intervención psicoterapéutica que han sido validados por la investigación, y se afirma la primacía del juicio clínico en el proceso de toma de decisiones en la práctica.

$3^{\circ}$ Los psicoterapeutas comprometidos en docencia en psicoterapia que buscan enseñar técnicas cuya eficacia haya sido validada empíricamente. Se describe como se deben usar en el entrenamiento de psicoterapeutas los datos obtenidos por la investigación empirica.

\section{Abstract}

This paper reviews critically how psychotherapy research may be of interest to:

1. Government authorities and health insurance industry, for the best planning of the psychotherapeutic service delivery system. Here the author puts readers on their guard against the misuse of psychotherapy research results.

2. Clinicians that seek to expand the evidence base of their therapeutic interventions. Relevance of "curative factors" validated by research and primacy of clinical judgement in the therapeutic decisionmaking process is stressed.

3. Psychotherapists engaged in training that intend to teach therapeutic techniques that have proved their efficiency. The way empirical research data should be used in the training of psychotherapists is

Key words: Psychotherpy, research, methodology.

\section{Introducción}

Es indudable que la investigación en psicoterapia llegó a las costas latinoamericanas y llegó para quedarse. Desde que en 1992 un grupo de psicoterapeutas nos reunimos en Mendoza para fundar el capítulo latinoamerciano de la SPR, el

campo de la investigación en nuestra región ha crecido en cantidad y en calidad. En estos 6 años, el interés por la novedad en la evaluación de la psicoterapia con metodologia empírica ha dado paso a una actitud más serena y reflexiva en relación con los alcances y logros que pueden esperarse.

${ }^{1}$ Conferencia dictadaen el $4^{\circ}$ Encuentro del Capitulo Sudamericano de la Society for Psychotherapy Research 27.9. 1998. Montevideo Uruguay

${ }^{2}$ Médico-Psiquiatra, Psicoanalista, Profesor Universidad de Chile 
En esta presentación quisiera hacer una revisión del interés y relevancia de la investigación para el desarrollo de la psicoterapia, en base a la experiencia que hemos ido acumulando en nuestros países, experiencia que ha sido elaborada a través de los contactos con colegas e instituciones de la región. Desde ya reconozco un sesgo en mi manera de ver las cosas, desde el momento en que mi compromiso con la psicoterapia está fuertemente influida por mi calidad de miembro activo de la Asociación Psicoanalítica Chilena, de académico del Departamento de Psiquiatria y Salud Mental de La Universidad de Chile y de miembro fundador del Comité de Psicoterapia de la Sociedad de Neurologia, Psiquiatría y Neurocirugía de Chile. En estas instituciones hemos desarrollado actividades docentes y de investigación, y colaborado con las autoridades gremiales y gubernamentales en la creación y establecimiento de políticas de atención en psicoterapia y de formación y perfeccionamiento continuado de psicoterapeutas y/o psicoanalistas. En las actividades académicas y profesionales me he movido en un ambiente pluralista, donde convivimos psicoterapeutas psiquiatras y psicólogos de distintas orientaciones, en especial sistémicos, cognitivoconductuales y psicoanaliticos. Junto con lo anterior, y como debe suceder con la mayoria de los que estamos acá reunidos, mi actividad principal es la práctica clinica, es decir, el trabajo con pacientes que acuden en busca de ayuda para mitigar su dolor psíquico, para reducir sus sintomas y para encontrar salidas a los laberintos en que la vida los ha situado. Así, actualmente me defino como un clínico cuya relación directa con la investigación empirica es bastante menor que el contacto indirecto con ella a través de la literatura. Básicamente, soy un clínico lector-¿ ¿o debiera decir "consumidor"?-de literatura especializada e interesado en los procesos de aplicación de ésta a la práctica y a la enseñanza de la psicoterapia.

En el contexto descrito, surgen naturalmente tres campos de aplicación de la investigación en psicoterapia que deben ser considerados cuidadosamente. El primero es el estudio de la atención psicoterapéutica dentro del sistema general de aten- ción en Salud. En este punto, la investigación en psicoterapia se encuentra con los problemas y enfoques propios de la Epidemiologia, de la Salud Mental y de la interfase entre Economía y Salud Pública.

El segundo campo de aplicación es el de la clínica psicoterapéutica, indudablemente el que más interés despierta en nosotros y que constituye el punto de partida y el corazón mismo de la investigación empírica. Aquí, los tópicos se resumen en los conceptos de investigación en proceso psicoterapéutico y sus resultados, cuya meta natural se orienta a definir los factores de cambio en la relación terapéutica, los llamados factores curativos; la idea central es que el aislamiento de los "ingredientes activos" irá necesariamente en favor de un mejoramiento de la técnica psicoterapéutica. Como investigación clínica sofisticada, la investigación en proceso y resultado se ha ido deslizando en los ultimos años hacia un nuevo horizonte,-más problemático y ambicioso-, y que se resume en la expresión de "práctica psicoterapéutica basada en la evidencia". Esta manera ultramoderna de entender las cosas, pretende estandarizar y regular la práctica clínica desde el punto de vista del sistema de atención en salud y acerca el campo psicoterapéutico a la "medicina basada en la evidencia".

Un tercer campo de aplicación de la investigación en psicoterapia se refiere a la formación de psicoterapeutas. Si hemos ido acumulando un corpus de conocimientos relativos a los factores curativos, la pregunta que sigue es acerca de la posibilidad de diseñar técnicas de enseñanza que maximicen el aprendizaje de los mismos. En algunos países de nuestra región, como por ejemplo en Chile, éste es un tema candente desde el momento en que asistimos a una explosión no regulada de la oferta de profesionales, en especial de psicólogos. La liberalización de la educación superior ha traido por consecuencia la apertura de decenas de nuevas escuelas de psicología, que anualmente están entregando al "mercado psicoterapéutico" -acostumbrémonos a la terminología-, una cantidad de recursos humanos que sobrepasa con creces la po- 
sibilidad de entrenamiento mínimo en psicoterapia. Hay creciente consenso en que los estudios de pregrado no capacitan a los psicólogos para desempeñarse como psicoterapeutas; esto también es cierto en el caso de los psiquiatras: la especialización entrega sólo rudimentos de capacitación psicoterapéutica. El problema es que no existe capacidad en las instituciones de postgrado, públicas o privadas, para formar en psicoterapia-no importando la orientación que prefiramos-, a tanto psicólogo y psiquiatra principiante.

Un cuarto campo de investigación en psicoterapiay que en nuestra región por el momento prácticamente no tiene aplicación, es el de la investigación básica, pues ésta depende de estructuras universitarias altamente desarrolladas y de niveles de financiamiento público inexistentes. Por investigación básica entiendo la investigación que articula la pregunta por el cambio terapéutico con otras disciplinas cientificas, como las ciencias cognitivas, la lingüística, la teoria de la comunicación, la informática, la relación temprana madre-hijo, etc.

\section{Epidemiologia, SaludMentale Investigación en psicoterapia}

Para los clínicos es dificil darnos cuenta de que nuestra práctica psicoterapéutica está inserta en un sistema de atención en Salud Mental, que a su vez es parte de una sociedad con valores, creencias e instituciones culturales que determinan concepciones y expectativas diferentes frente a los procesos de enfermary mejorarse mentalmente. Mientras la psicoterapia era una actividad artesanal que se regía por economias de escaso impacto social, los clínicos podiamos mantener la ilusión de ser profesionales liberales con la posibilidad de establecer contratos de tratamiento con personas particulares, sin ingerencia de terceras partes. Sin embargo, en el contexto de una economía globalizada y en una cultura de modernidad tardía, en la que las dudas existenciales sobre el sentido de la vida y la propia identidad son fuente creciente de psicopatologia, la psicoterapia se ha convertido en un objeto de consumo masivo. No es por casualidad que, hace un par de años, el ConsumerReport-antigua institución americana de investigación de mercado-eligiera la satisfacción con la psicoterapia como objeto de encuesta a miles de consumidores estadounidenses. Por otra parte, la preocupación planteada a los gobiernos (en especial en USA, Gran Bretaña y Alemania) por el aumento desenfrenado del gasto en salud también alcanza a los profesionales de la psicoterapia, a quienes las instituciones de financiamiento en salud empiezan a exigir responsabilidad por el manejo de los tratamientos, de acuerdo con reglas que maximicen la relación costo-beneficio y cuya eficacia esté basada en criterios estrictos de evaluación empírica (Empirical Supported Treatments, ESTs). En los Estados Unidos, la intervención de la industria de seguros de salud en la intimidad de los consultoriosavalada por leyes federales-, ha llevado a una gran crisis en la práctica psicoterapéutica que no es el caso de describir acá.

En nuestros países, especialmente en los del Cono Sur, la preocupación de los Gobiernos por estos temas crece día a dia y más vale estar preparados para las negociaciones que ya se han hecho presentes y para las que se avecinan. Aquí surge una primera y urgente necesidad de investigación en nuestra región. Un interés principal de las autoridades es la relación entre psicoterapia y economía.

Sabemos que el tema del impacto económico de la psicoterapia es un asunto aclarado hace ya tiempo. La revisión más reciente es la hecha por Gabbard y cols. (1997), en la que después de seleccionar 686 estudios publicados en revistas de habla inglesa que tuvieran como objetivo evaluar si una intervención psicoterapéutica (individual, de grupo o familiar) en enfermedades psiquiátricas tuvo alguna implicación en los costos de atención, concluyen que la psicoterapia reduce los costos generales tanto en pacientes esquizofrénicos, bipolares y con trastornos borderline de personalidad. El trabajo de Gabbard y cols. de 1997 fue publicadoy comentado en el primer número de la flamante revista "Evidence Based MentalHealth" de febrero de este año (1998), bajo el título de "La psicoterapia reduce una variedad de costos 
cuando se usa para tratar pacientes con los desórdenes psiquiátricos más severos".

Este tipo de estudios corresponde a lo que Meyer (1991) llamó la "etapa de justificación" de la psicoterapia, que comenzó como respuesta a la publicación de los estudios de Eysenk en 1952 en los que este autor puso en duda la eficacia de los tratamientos psicológicos en comparación con la mejoría por remisión espontánea. En nuestros países, estamos lejos de haber completado las tareas de la "etapa de justificación". Recientemente, unos colegas de un país vecino me pidieron asesoria para iniciar un estudio comparativo de costo-beneficio sobre el impacto de la psicoterapia en pacientes con comorbilidad somática. La intención de ellos es, precisamente, justificar frente a las autoridades el pago de psicoterapias por los seguros de salud. Es probable que necesitemos muchos estudios de este tipo en nuestros países que repliquen investigaciones extranjeras ya realizadas. Un estudio así tiene una influencia totalmente diferente a una mera referencia bibliográfica. Sin embargo, investigadores y clínicos debemos tener claro que tales estudios son de doble filo. Por cierto, podremos convencer a las autoridades de que la psicoterapia sí es eficaz, que lo es más que el placebo y que la remisión espontánea, y que reduce el gasto general en salud en pacientes con comorbilidad; incluso podremos convencerlos de que la psicoterapia forma parte de la actualmente llamada "prevención primordial", cuyo objetivo es aumentar las conductas personales y sociales de autocuidado en salud. Si logramos mostrar una relación costo-beneficio positiva, los seguros de enfermedad ciertamente cubrirán los tratamientos psicoterapéuticos. Pero, estos mismos estudios también darán argumentos a las autoridades para reglamentar la práctica psicoterapéutica, pues con seguridad redescubriremos en nuestros servicios hospitalarios y consultorios la misma curva de adherencia al tratamiento psicoterapéutico que se ha descrito en el resto del mundo.

Se sabe que la mayoria de los pacientes psicoterapéuticos reciben una cantidad relativa- mente pequeña de sesiones de terapia. En los Estados Unidos, se estima que la duración promedio de la psicoterapia es de 5 a 8 sesiones (Howard y cols. 1992; Garfield 1986; Phillips 1985). Los estudios sobre la utilización de los servicios del sistema de atención psicoterapéutica revelan la paradoja de que la mayoría de los pacientes acuden a un número relativamente pequeño de sesiones, mientras que la mayor parte del tiempo disponible de los psicoterapeutas es empleado en tratamientos de larga duración. Mientras más experiencia y años de profesión tengan, los psicoterapeutas atienden una muestra cada vez más restringida y seleccionada de pacientes, a los cuales les dedican mayor tiempo. La curva de adherencia al tratamiento psicoterapéutico (Phillips 1988 y 1992), universal e inmodificable en sus características esenciales, muestra que después del primer contacto cerca de un $50 \%$ de los pacientes abandona el tratamiento. Entre el segundo y el tercer contacto, entrevista o sesión de terapia, un $10 \%$ deja de asistir. Después de cada una de las sesiones sucesivas, se pierde alrededor de un 10\% adicional de los pacientes. Así, se determina una curva de decaimiento rápido hasta llegar a una asintota. Cifras comparables han sido halladas en tratamientos psicoterapéuticos realizados en consultorios privados de acuerdo con investigaciones realizadas en California (véase Messer \& Warren 1995, pp.6s) $)^{3}$. Salta a la vista que el paciente psicoanalitico típico pertenece a este tipo de pacientes "crónicos". La "historia natural" del paciente psicoanalítico suele revelar el paso previo por psicoterapias de duración variable antes de llegar a tenderse en el diván. Se da así una asociación natural entre psicoterapeuta principiante y terapia breve. El terapeuta experto, en cambio, dedica la mayor parte de su tiempo a psicoterapias prolongadas con pacientes que sufren trastornos de personalidad (eje II).

Este panorama epidemiológico ha llevado a las autoridades a favorecer el financiamiento de tratamientos psicoterapéuticios breves, pues éstos cubririan las necesidades de más del $80 \%$ de la pobla- 
ción consultante. Este es por lo demás un criterio general de Salud Pública -el favorecer tratamientos de amplia cobertura-, que ahora está siendo aplicado por las agencias de financiamiento de psicoterapias. Como ya sucede en países del Hemisferio Norte, los tratamientos largos y de alta frecuencia, como el psicoanálisis, tendrán crecientes dificultades para ser financiados por los seguros. Esta politica afecta en especial a los terapeutas expertos, o sea, a los líderes cientificos y gremiales y a sus pacientes. De ahí que desde hace algunos años aumenta el interés de los psicoterapeutas en investigar la relación costo-beneficio en tratamientos de larga duración.

Probablemente muchos estarán en este momento pensando que la situación descrita no se da en nuestro medio, desde el momento en que el Estado Benefactor, con sus garantias de seguros de enfermedad, nunca ha llegado a ser dominante en estos pagos. Sin embargo, si apostamos al desarrollo económico de la región, no existen razones para pensar que el problema no se hará presente también acá.

Por otra parte, dada la diversidad en la oferta de tratamientos psicoterapéuticos, las autoridades deben decidir qué tipo de tratamientos favorecer a través del financiamiento, pues es evidente que la oferta psicoterpaútica es extremadamente poco transparente: ¿es el público capaz de diferenciar entre expertos y charlatanes? Aquí nos encontramos nuevamente con problemas, pues sólo algunas orientaciones terapéuticas pueden mostrar evidencias empíricas sobre su eficacia. En la práctica, actualmente sólo las terapias cognitivoconductuales cumplen con los estrictos criterios que está exigiendo la llamada "práctica basada en la evidencia" para considerar que un tratamiento ha probado cientificamente su eficacia ${ }^{4}$. Por cierto, estos criterios son muy discutibles y varian de país en país, por ejemplo, son más estrictos en USA que en Alemania. En nuestros países estamos aún lejos de esta controversia, pues recién empieza a discutirse a nivel de autoridades ministeriales la necesi- dad de regular el ejercicio psicoterapéutico. Para ello, por supuesto se requiere información que debe surgir de investigaciones epidemiológicas.

Durante los años 1992-93 estudiamos en Chile el sistema de atención psicoterapéutica (Jiménezy cols. 1994, 1995). Usando una metodología cualitativa intentamos responder dos preguntas básicas. La primera fue "¿cómo se llega a ser paciente psicoterapéutico?" y la segunda “¿cómo se llega a ser psicoterapeuta"? El principal resultado de esa investigación fue comprobar la casi total ausencia de datos que permita la aplicación de politicas racionales en la asignación y distribución de recursos humanos y financieros y en el entrenamiento de psicoterapeutas. También fue claro que la industria de seguros de enfermedad toma sus decisiones principalmente en base a estudios de mercado, tratando de maximizar las ganancias, lo cual no necesariamente va en beneficio de los pacientes y de sus terapeutas. La situación chilena creo es extrapolable al resto de Latinoamérica. En nuestra región no existen estudios que permitan definir el perfil de la población consultante (demografia, diagnóstico, filtros en la "carrera de paciente", etc.), ni tampoco las características de los psicoterapeutas (demográficas, distribución, carrera profesional, etc.). Desde luego, tampoco existen estudios de proceso y resultado nide impacto de las psicoterapias en el gasto en salud.

A propósito de esta investigación, realizamos un seminario sobre el tema "financiamiento de las psicoterapias", al que invitamos a representantes de las compañias de seguros de salud, con la idea de motivarlos a entregar la información que les sería requerida por los encuestadores. Sabíamos que esto podía modificar la situación a estudiar pero lo que nos sorprendió fue la rapidez con que aquello sucedió: a las pocas semanas las compañias cuyos representantes habian asistido al seminario habian decidido reducir drásticamente la oferta de financiamiento de psicoterapias. Sólo tomaron aquellos datos que favorecían el ahorro inmediato en las prestacio-

\footnotetext{
${ }^{4}$ Mayor información sobre la llamada "controversia de los tratamientos con apoyo empírico" (Empirically Supported Treatments Controversy) puede encontrarse en el vol 8. número 2 de la revistaPsychotherapy Research (1998).
} 
nes, sin considerar las evidencias -que también mostramos-, en torno a los requisitos formativos del psicoterapeuta que pueden garantizar una práctica ética y técnicamente óptima. Actualmente muchas de estas instituciones de seguros ofrecen atención cerrada y -en ausencia de una reglamentación legal sobre el ejercicio de la psicoterapia-, contratan para ello profesionales recién egresados con escasa o ninguna formación psicoterapéutica. Situación que, me he enterado, es análoga en Argentina. Al bajar de este modo los costos de la atención, ciertamente se maximiza la relación costo-beneficio, pero no del tratamiento, sino de las compañias aseguradoras.

Lo que me interesa trasmitir en este punto es que los clinicos interesados en investigación, especialmente aquellos que además tienen alguna responsabilidad en la formulación de politicas en Salud Mental o que ejercen asesorias a las autoridades, deberían aprender a leer de manera crítica la literatura disponible. Cómo veremos, esto es igualmente verdadero cuando se trata de integrar los hallazgos de la investigación a la práctica clínica. El juicio clínico es un complejo proceso de toma de decisiones y los hallazgos empíricos son sólo una fuente más -por importante que ella sea-, de fundamentación de una práctica óptima. No es tarea simple poner de acuerdo los intereses de los pacientes, con los de los terapeutas y éstos con los de las autoridades de Salud y de la industria de seguros de enfermedad.

La experiencia internacional enseña que existe el peligro real de que los hallazgos de la investigación empírica en psicoterapia sean usados acríticamente por las autoridades como un recetario para planificar y regular la práctica psicoterapéutica. En la medida en que la evidencia que surge de la investigación empieza a formar un cuadro coherente, aumenta la tentación de convertir estos hallazgos en respuestas definitivas, sin considerar la extrema cautela con que los propios investigadores los entregan a la consideración de la comunidad cientifica (Roth \& Fonagy 1996).

\section{3. ¿Qué tipo de datos empiricosson relevantes parala práctica clinica?}

El hiato entre investigación empírica y clínica psicoterapéutica, reiteradamente denunciado, muestra la complejidad de la relación entre investigación y clínica. El campo de la investigación en psicoterapia se ha transformado en un campo de batalla donde luchan intereses contradictorios de diferente tipo. Ya hice alusión al uso (y abuso) que las autoridades de salud y la industria de seguros de enfermedad hacen de los hallazgos empíicos. Ahora quisiera referirme al interés que tiene la investigación empírica para la práctica clínica y la docencia en psicoterapia.

Si bien manifiestamente investigadores y clinicos persiguen el mismo objetivo, cual es el mejoramiento de las técnicas de tratamiento, se ha dicho que esta coincidencia es más aparente que real (Roth \& Fonagy 1996, pp. 41s). Mientras la investigación pretende hacer evidentes las relaciones causales entre intervenciones y mejoría mediante la aplicación de controles metodológicos, la actitud del clínico es mucho más pragmática: le interesa menos la demostración de cuál elemento es el ingrediente activo que el logro del cambio en sí mismo. En este sentido, se puede decir que el investigador busca maximizar la evidencia negativa, es decir, aumentar el nivel de cuestionamiento y crítica de los hallazgos, mientras que el práctico hace lo contrario: maximiza la evidencia positiva para poder actuar coherentemente en la situación clinica (Bowlby 1979).

En mi experiencia, sin embargo, esta divergencia, que aparece como tan radical, no se da en la realidad de la manera que podría desprenderse de lo dicho anteriormente. Más aún, durante los años en que estuve en contacto estrecho con actividades de investigación y con investigadores a tiempo completo (1985-1990) en la Univeridad de Ulm, observé en mi propia práctica con pacientes un efecto absolutamente contrario al esperado. Después de un corto período en que las convicciones clínicas adquiridas durante mi formación psicoterapéutica 
y psicoanalitica en Chile se vieron sometidas a un cuestionamiento sistemático por el ambiente de intensa crítica que existía en el departamento de psicoterapia en que trabajaba, y durante el cual me invadieron intensos sentimientos de incertidumbre y contradicción, empezé rápidamente a darme cuenta de los notables cambios que se produjeron en múltiples dimensiones de mi actividad clínica. Estos cambios coinciden con lo que Safran y Muran (1994) han descrito como "productos clínicos secundarios a la investigación".

Lo primero es el nacimiento de una nueva "actitud empirica", esto es, de un hábito de evaluar las propias teorías a la luz de los fenómenos observados más que de seleccionar los eventos con el objeto-desde luego, preconsciente-de fundamentar la propia teoria. No puedo profundizar mucho en este complejo problema de epistemología clínica. Sólo adelantaré mi opinión de que el método clínico tradicional, representado en este caso por el método psicoanalitico, no entrega herramientas suficientes para distinguir lo ideológico de lo científico en la práctica psicoterapéutica. En contra de lo esperado inicialmente, la introducción del análisis didáctico no disminuyó la influencia de la ecuación personal o de las ideologias en la construcción de teoria en psicoanálisis (Ferenzci 1928, Jiménez 1994); sucedió más bien que el mismo análisis didáctico sufrió un proceso de idealización. Ya desde los tiempos de Freud el método psicoanalitico ha sido idealizado (Jiménez 1998), idealización que se rompe rápidamente cuando un clínico se compromete en actividades de investigación. En la Unidad de Psicoterapia Psicoanalítica del departamento universitario donde trabajo, los miembros del equipo docente regularmente conducimos entrevistas clinicas y psicoterapias detrás del espejo de visión unidireccional frente a los demás colegas y a los alumnos, psicólogosy psiquiatras principiantes que siguen un programa básico de formación. Todos estamos de acuerdo en que la exposición regular a la supervisión de los pares y de los propios alumnos tiene un gran efecto educativo en quienes nos sometemos a ella; es el mejor antídoto en contra de la idealización de los maestros, de las ideologías y de la adhesión acrítica a escuelas psicoterapéuticas. La experiencia de ver a psicoterapeutas expertos dando cuenta de su trabajo con el paciente deja en evidencia el hiato existente entre la práctica psicoterapéutica real y las idealizaciones de la misma que se trasmiten a través de los relatos clínicos tradicionales.

El aumento en la claridad conceptual es una segunda ganacia para los clínicos que se acercan a la investigación. La necesidad de operacionalizar los propios conceptos y de articular las evidencias en pro y en contra de las hipótesis de investigación contribuye decisivamente a acotar lo que podemos llamar la teoría clínica, en contraposición con el nivel de la teoria metapsicológica, más alejado de los fenómenos y por eso mismo más proclive a la inflitración no crítica de ideologías de escuela y orientaciones terapéuticas. Esta claridad conceptual acrecienta la capacidad de hacer explícitas las propias teorias clínicas implicitas (Sandler 1983), tarea indispensable para favorecer un diálogo productivo en el ámbito clínico.

Lo notable es que este cambio de actitud frente al trabajo clínico y a las propias teorías e ideologías terapéuticas, en vez de conducir a una suerte de paralización frente al paciente, produce exactamente lo contrario. En mi experiencia, aumenta la libertad para pensar con el paciente acerca de las intervenciones técnicas que mejor pueden ayudarlo. El pragmatismo, que podría conducir a una suerte de oportunismo teórico, se ve compensado con la necesidad de fundamentar racionalmente las intervenciones terapéuticas. Caspar (1998), investigando los procesos de procesamiento de información que tienen lugar en la mente del terapeuta, hace notar que los mejores resultados en el domino clínico de generación de hipótesis se logra con la combinación de procesos intuitivos y analitico-racionales. Estoy convencido que el entrenamiento en investigación facilita las mejores combinaciones en los procesos de información y de toma de decisiones en el nivel clinico.

Pero, para que se transforme en conocimiento útil, la investigación empírica debe ser integrada al juicio clinico. La cuestión crucial para la integración está 
en el tipo de investigación empírica que puede ser utilizada clinicamente. Hay un amplio consenso en que un gran porcentaje de la investigación existente carece de aplicabilidad en la práctica. La insistencia en probar la eficacia de ciertas técnicas, mediante metodologias que buscan la estandarización de las poblaciones, debilita la posibilidad de generalización y de validez ecológica de los resultados; el aumento en validez interna se hace a costa de una disminución de la validez externa. Por lo demás, hay datos empiricos que sustentan la idea de que aún en estudios en que mucho de la varianza está determinada por conceptos prescriptivos (técnica), una considerable parte de la misma la determinan los terapeutas individuales. Lambert, Shapiro y Bergin (1986) concluyen que no más del $15 \%$ de la varianza de los resultados es debida a factores específicos, como la técnica empleada, mientras más del $45 \%$ se explica por factores no específicos, tales como las expectativas de los pacientes de que la terapia los ayudará (alianza terapéutica).

En este punto, quiero relatar una experiencia concreta. En el Comité de Psicoterapia de la Sociedad de Psiquiatría hacemos reuniones regulares para discutir artículos de investigación que pueden tener interés para terapeutas de distintas orientaciones. Este año uno de los temas ha sido el de la alianza terapéutica. En una reunión reciente se discutió el trabajo "Dimensiones de la alianza terapéutica y sus asociaciones con el resultado en terapia familiar" de Quinn y cols. (1997). Este trabajo, en resumen, reporta el hallazgo de que cuando la alianza con las esposas es más fuerte que la alianza con los esposos, el resultado es más positivo que cuando se da la situación contraria. Los autores terminan recomendando que los terapeutas familiares deben enfatizar la alianza con las mujeres, en especial al comenzar el tratamiento. De inmediato se produjo una agitada discusión lidereada por los terapeutas familiares presentes en la reunión. El argumento principal se centró en torno a la afirmación de que el terapeuta familiar debe, precisamente, mantener una equidistancia en las alianzas con los distintos miembros de la familia o pareja. Finalmente se llegó al consenso de que este hallazgo, más que una prescripción (que fue como lo presentaron los autores), debía tomarse como un indicador pronóstico, en el sentido de que la comprobación de una buena alianza con la mujer ofrece un terreno firme para trabajar los conflictos entre los miembros de la familia. Esto es distinto que afirmar que debe buscarse establecer una alianza con las mujeres. En la discusión quedó en evidencia que los hallazgos de investigación empírica sólo pueden ser utilizados cuando "hacen sentido" clínico.

La calidad del juicio clínico del terapeuta contribuye grandemente al resultado de la terapia: los terapeutas necesitan desarrollar una visión realista de sus pacientes, en términos de los recursos disponibles y de las restricciones existentes tanto en el nivel de los conflictos del paciente como en el del establecimiento y mantención de la relación terapéutica y, desde alli, deben construir el procedimiento terapéutico más adecuado. Esa visión coincide con la planteada por algunos psicoanalistas como Greenson (1960), Bowlby (1969) y Perterfreund (1975) para quienes el analista construye, en su trabajo día a día con su paciente un "modelo de trabajo" que reúne los distintos aspectos y rasgos de aquél. Para Greenson, el modelo de trabajo es un "tipo especial de representante de objeto interno. Es una representación interna que no está fundida con el selfy que, sin embargo, no es ajena a él. Invistiendo el modelo de trabajo, como un suplemento del paciente externo, uno se acerca a los procesos identificatorios empáticos" (Greenson 1960, p.423). La escucha empática a través del modelo de trabajo es una función del yo experiencial del terapeuta. Los programas y la información almacenada que constituyen los diferentes modelos de trabajo representan selecciones específicas de la información total disponible por el terapeuta a lo largo del tiempo. Así, el modelo de trabajo es el resultado de la experiencia concreta del terapeuta con este paciente en particular, por una parte, y toda la información teórica y la experiencia práctica que el terapeuta ha acumulado a lo largo del tiempo, por el otro. Los modelos de trabajo son mini-teorias en acción, teorías en su referencia concreta a la interacción actual. 
Esta manera de entender los procesos de procesamiento de información y de toma de decisiones del terapeuta, compatible con la poca investigación empírica existente en este dominio (véase Caspar 1988), nos permite entender mejor la manera indirecta y contextual en la que los clinicos podemos utilizar los hallazgos empíricos. Es claro que los estudios empíricos entregan perfiles de pacientes promedios que no representan a ninguna persona actualmente existente; además, usan instrumentos de evaluación que tampoco reflejan la manera clinica de acercarce a los fenómenos. Sin embargo, Maling \& Howard (1994) hacen notar que la mente del clinico trabaja con el supuesto de que hay clases o grupos de pacientes que responden de manera similar a las mismas intervenciones técnicas. Lo que se sugiere entonces, es que los hallazgos empiricos pueden organizarse en torno a prototipos, semejantes a las clases con las que trabaja el clinico. De este modo, la información empírica puede ser usada por el terapeuta práctico.

Sin embargo, la organización de la información empírica "cruda" en "prototipos útiles en el proceso de construir un procedimiento terapéutico individualizado para cada nuevo paciente y para cada nueva situación" (Caspar 1997 p.107), pasa por la construcción de modelos teóricos. Los modelos asi concebidos, son una forma "blanda" de estandarización, a diferencia de la forma "dura", preconizada por el movimiento de tratamientos basados en la evidencia, que pretende encontrar procedimientos específicos y cuasi algorítmicos para cada trastorno delDSM IV. Son estos modelos heurísticos, como interfase entre la investigación y la clinica, los que pueden ser enseñados en los programas de formación de psicoterapeutas. Hay ciertos manuales de psicoterapia dinámica, como el de Luborsky (1984) y el de Strupp (1984), que cumplen con esta función de servir de guia estratégica y que integran sabiduria clinica y hallazgos empiricos.

En Chile, De la Parra y Cols. (1998) investigaron la utilidad del manual de Struppy cols.(1984) "Guía para la psicoterapia dinámica de tiempo limitado" (TLDP), en la enseñanza de la psicoterapia a tera- peutas principiantes. Encontraron la misma discrepancia referida por Henry y cols. (1993) en la aplicación del manual a terapeutas expertos en el estudio Vandelbilt II, en el sentido de que la adherencia al mismo no asegura el éxito terapéutico. Sin embargo, a diferencia de estos autores, De la Parra y cols. concluyen que sus terapeutas inexpertos paradójicamente no parecieron aprender estrategias especificas con el manual, sino que más bien aprendieron a manejar procesos interpersonales de manera de mantener una alianza terapéutica adecuada para el éxito.

Sin embargo, a mi entender, el caso más logrado en este sentido es el del modelo genérico de Orlinsky y Howard $(1986,1987,1994)$. En su versión más reciente, Orlinsky y cols. (1994) tabularon un total de 2.343 hallazgos independientes de relaciones proceso-resultado. Para transformar esta masa de datos dentro de un cuerpo coherente de conocimientos se requiere de un modelo teórico de terapia capaz de organizar las muchas y distintas variables en un conjunto pequeño pero abarcativo de categorias conceptuales. Así se desarrolló el modelogenérico.

Del modelo genérico me interesa destacar acá la estrecha relación que éste asigna al impacto de las intervenciones técnicas del terapeuta, la disposición del paciente para recibirlas y la calidad del vínculo entre ambos. El monitoreo de la interacción de estos tres aspectos en las sesiones de terapia son de gran utilidad no sólo en el propio trabajo como terapeuta, sino también en las supervisiones de casos en psicoterapia y psicoanálisis. El modelo genérico postula que la participiación del paciente en las intervenciones técnicas es crucial en el resultado del tratamiento. Los predictores más fuertes en este sentido son la capacidad del paciente de colaborar versus de resistirse al trabajo terapéutico, junto con la experiencia del paciente de afectos positivos hacia el tratamiento y la persona del terapeuta. La calidad del vinculo se expresa en dos series de fenómenos: la primera es la fortaleza del equipo de trabajo, es decir, la investidura personal de ambos participantes y la habilidad de coordinar la acción en sus respectivos roles. La otra es la calidad de su 
relación personal, esto es, la resonancia de la comunicación mutuay los afectos que cada uno elicita en el otro.

Durante el último tiempo he hecho la experiencia de supervisar casos de análisis guiado por las categorías de proceso que ofrece el modelo genérico. Hay distintas tradiciones en los estilos de supervisión analiticas. Personalmente sigo las recomendaciones de Szecsödy (1990), basadas en investigaciones empíricas. Se trata de crear en la relación con el supervisando una situación de "aprendizaje mutativo", en la que éste aprenda a reconocer el sistema de interacción que establece con su paciente. En este marco, sugiero a los supervisandos revisar el estado del vínculo terapéutico antes de empezar a intentar dilucidar, por ejemplo, las características de la fantasia transferencial inconsciente actuante en esa sesión. Un ejemplo puede aclarar mejor lo que quiero decir.

Se trata de un candidato avanzado en su formación analitica que supervisa un segundo caso. $\mathrm{Su}$ paciente, un joven de 21 años, estudia derecho y consulta por ideación obsesiva (temores irracionales a tener sida), angustia difusa, timidez, dificultades en el contacto interpersonal y miedos homosexuales. El inicio del análisis fue dificil, con un fuerte despliegue de defensas obsesivas, envueltas en una situación de "no tocarse", que fue rápidamente caracterizada como "estar dentro de un tubo". Las asociaciones giraban casi exclusivamente en torno a los sintomas obsesivos y no habia referencia a otras situaciones o afectos en relación con terceros o con el analista. Por su parte, el analista sentía que sus interpretaciones iban a quebrar al paciente y notaba que la excesiva cautela de su parte aumentaba la ansiedad del paciente.

El monitoreo del vínculo de acuerdo con las categorías del modelo genérico, nos llevó rápidamente a detectar problemas en elárea de la coordinación interactiva. El paciente se refugiaba en un tipo de pasividad controladora y el terapeuta se veía envuelto en una actitud que permitía este control. La sugerencia de interpretar directamente esta situación, condujo en un primer momento al "quiebre" del tubo, que se manifestó concretamente en el pa- ciente a través de un ataque de pánico durante un fin de semana.

El trabajo interpretativo en este área permitió a la diada paciente analista salir progresivamente del "tubo", con lo cual el material se hizo más emocional, primero, en referencia con relaciones externas (su familia y su novia), posteriormente, con el analista mismo.

Después de un período en que se consolidó el "equipo de trabajo", en el monitoreo de acuerdo con el modelo genérico otros aspectos de la relación empezaron a tener importancia. Por ejemplo, hubo largos períodos en que el problema más importante lo encontrábamos en el área de la resonancia empática. El analista se sentía aislado de su paciente, el material no le hacía sentido. Parecía que el "tubo" se habia trasladado a la contratransferencia del analista. Precisamente, la reflexión sobre esta (contra)resistencia, que llevó a descubrir ciertas fantasias contratransferenciales, permitió que el proceso siguiera adelante. Más adelante, el analista notó la aparición de sentimientos positivos en relación con su paciente, lo que, de acuerdo con el modelo, fue entendido como un indicador favorable de proceso analítico. En fin, no pretendo hacer un relato completo, sólo quiero ilustrar el uso de las categorías del modelo genérico para monitorear desde un punto de vista "formal", el estado, sesión a sesión, del vinculo terapéutico. La concepción que hay detrás de esta manera de supervisar, hace justicia al enorme cúmulo de evidencias empíricas y al creciente consenso clínico de que la calidad del vinculo terapéutico es el factor curativo más poderso en psicoterapia. La idea es que las resistencias y contrarresistencias, provenientes básicamentes de la interacción de transferenciay contratransferencia, subvierten permanentemente el "mejor vínculo" posible entre terapeuta y paciente. Las categorias del modelo genérico permiten detectar el problema rápidamente para asi poder superarlo a través de intervenciones adecuadas.

Debo terminar acá, sin referirme a otras categorías del modelo genérico que también tienen un gran valor heurístico en psicoterapia. No dije nada sobre otros modelos notables y su aplicación en la 
clínica. Por ejemplo, las formalizaciones de caso que diversos investigadores han desarollado, como el CCRT de Luborsky o el Análisis Configuracional de Horowitz, entre otros. Tampoco me extendien el importante tópico de la investigación en entrenamiento en psicoterapia, que por lo demás es bastante escasa: si ni siquiera tenemos evidencia de hasta qué punto el principal factor curativo, esto es, la capacidad de establecery mantener la alianza terapéutica, puede ser entrenado (Luborsky 1993) .

Con estas reflexiones espero haber contribuido a que el hiato entre investigación y clínica se haga más pequeño en Uds. e incentivarlos a trabajar para presentar una investigación empírica más "amable" a los colegas clinicos que siguen desconfiando de sus ventajas.

\section{Bibliografia}

BowlbyJ (1979) Psychoanalysis as art and science. Int.Rev.PsychoAnal.6:1-14.

Bowlby J (1969) Attachment and loss vol I: Attachment. New York: Basic Books.

Caspar F (1997) What goes on in a psychotherapist's mind? PsychotherapyResearch7(2):105-125.

Dela ParraG, RumiallóP, \& Isla R (1998) The experience oftraining novel therapists with a dynamic $p$ sychotherapymanual: What do they reallylearn?(Manuscrito).

Ferenczi S. (1928) La elasticidad de la técnica. En: Problemas y métodos del psicoanálisis. Paidós, Buenos Aires, 1966, pp.77-88.

Gabbard G O, Lazar S G, HornbergerJ etal. (1997) The economic impact of psychotherapy: a review. AmJ Psychiatry 154: 147-55

Garfield S.L. (1986) Research on client variables in psychotherapy. En: S.L. Garfield \& A.E. Bergin (eds.), Handbook of psychotherapy and behaviorchange, 3rd edition. John Wiley. New York.

Greenson R (1960) Empathy and its vicissitudes. Int.J.Psycho-Anal. 41:418-424.

Henry WP, Strupp H H, Schacht T E \& Binder J L (1993) The effects of training in time-limited dynamic psychotherapy: Changes in therapist behavior. Journal of Consulting and Clinical Psychology 61:434-440.

Howard K I, Vessey J T, Lueger R, \& Schank D. (1992) The psychotherapeutic service delivery system. PsychotherapyResearch 2(3):164-180.
J iménezJ P (1994) El psicoanalista como pensador artesanal: Una exploración en los procesos mentales del analista. En: The analist's mind: from listening to interpretation. International Psychoanalytical Association. London pp.53-65.

J iménezJ P (1995) Un modelo de análisis del sistema de atención de psicoterapia en Chile. En: J P Jiménez, CBuguñá \& A Belmar (Eds.) Investigación en Psicoterpia. Procesos y Resultados. Investigaciones empíricas 1993-1994. Santiago: SPR (Capitulo Latinoamericano) \& CPU.

J iménezJ P(1998) El método clínico, los psicoanalistas y la institución. Presentado en el XXII Congreso de FEPAL, Cartagena de Indias, Colombia. Agosto 1998.

J iménezJ P, Florenzano R, BuguñáC, SarnoffR, VegaS (1994) El sistema de servicios de atención de psicoterapia en Chile frente a los desafios del presente y del futuro. Estudios Sociales 79 (1):85-115

Lambert M, Shapiro D, \& Bergin A (1986). The effectiveness of psychotherapy. En A E Bergin \& S L Garfield (Eds.) Handbook of psychotherapy and behavior change (pp. 157-212) New York: Wiley.

Luborsky L. (1984) Principles of psychoanalytic psychotherapy, A manual for supportive-expressive treatment. New York: Basic Books.

Luborsky L. (1993) How to maximize the curative factors in dynamic psychotherapy. En: NE Miller, L Luborsky J P Barber, J PDocherty Psychodynamictreatmentresearch. A handbook for clinical practice. Basic Books New York pp.520-535.

Maling M S \& Howard K I (1994) From research to practice to research to ... En: P F Talley, H H Strupp \& S F Butler (Eds.) Psychotherapy research and practice. Bridging the gap. Basic Books New York pp.247-253.

Messer S B, \& Warren C S (1995) Models of briefpsychodynamic therapy: A comparative approach. New York London: Guilford Press.

Meyer A E, Richter R, GraweK, Graf v.d. SchuldenburgJ M Schulte B (1991) Forchungsgutachten zu Fragen eines Psychotherapeuten Gesetzes. Im Auftrag des Bundesministeriums für Jugend, Familie, Frauen und Gesundheit. Universitäts-Krankenhaus Hamburg-Eppendorf.

Orlinsky DE (1994) Research-based knowledge as the emergent foundation for clinical practice in psychotherapy. En: P F Talley, H H Strupp \& S F Butler (Eds.) Psychotherapy research and practice. Bridging the gap. Basic Books New York pp.99-123.

Orlinsky DE \& Howard KI (1986) Process and outcome in psychotherapy. En A E Bergin \& S L Garfield (Eds.) Handbook of psychotherapy and behaviorchange (3rd ed.) New York: Wiley.

Orlinsky DE \& Howard KI (1987) Ageneric model of psychotherapy. Joumal of Integrative and EdecticPsychotherapy. 6: 6-27.

Orlinsky DE, GraweK \& Parks B K (1994) Process and outcome in psychotherapy - noch einmal. En A E Bergin \& S L Garfield (Eds.) Handbook ofpsychotherapy and behaviorchange (4th ed.) New York: Wiley. 
Peterfreund E. (1980) On information and systems models for psychoanalysis. IntRev. Psychoanal. 7:327-345.

PhillipsE L(1985) Psychotherapy revised: New frontiers in research and practice. Hillsdale, N.J.: Lawrence Erlbaum Associates Publishers.

PhillipsE L (1988) Patient compliance. New lighton health systems in medicine and psychotherapy. Toronto Lewiston NYBern Stuttgart: Hans Huber.

Phillips E L (1992) George Washington University's International Data on Psychotherapy Delivery systems: Modeling new approaches to the study of therapy. En: Larry E. Beutler \& Marjorie Crago (Eds.) Psychotherapy Research. An international review of programmatic studies. American Psychological Association. Washington.

Quinn W H, Dotson D, \& J ordan K (1997) Dimensions of therapeutic alliance and their associations with outcome in family therapy. PsychotherapyResearch7(4):429-438.
Roth A \& Fonagy P (1996) What works for whom? A critical review ofpsychotherapy research. NewYork: Guilford.

Safran J D \& Muran J Ch (1994) Toward a working alliance between research and practice. En: P F Talley, H H Strupp \& S F Butler (Eds.) Psychotherapy research and practice. Bridging the gap. Basic Books New York pp.206-226.

Sandler J (1983) Reflection on some relations between psychoanalytic concepts and psychoanalytic practice. Int. J. PsychoAnal. 64: 35-45.

Szecsödyl (1990) The learning process in psychotherapy supervision. Stockolm: Department of Psychiatry, Karolinska Institut. S:t Görans Hospital.

Strupp H H \& Binder J L (1984) Psychotherapy in a new key: A guide to time-limited dynamic psychotherapy. New York: BasicBooks. 